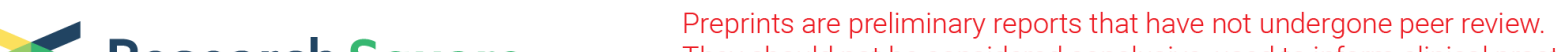 Research Square They should not be considered conclusive, used to inform clinical practice, or referenced by the media as validated information.
}

\section{Ga-DOTA-DiPSMA PET/CT Imaging: \\ Biodistribution, Dosimetry and First Comparison with 68Ga-PSMA-11 in Prostate Cancer}

Jiaying Zhang

First Affiliated Hospital of Fujian Medical University

Zefang Lin

First Affiliated Hospital of Fujian Medical University

Xiaojun Zhang

Key Laboratory of Radiopharmaceuticals, Ministry of Education, Beijing Normal University

Rong Lin

First Affiliated Hospital of Fujian Medical University

Mengchao Cui

Key Laboratory of Radiopharmaceuticals, Ministry of Education,Beijing Normal University

Jie Zang

First Affiliated Hospital of Fujian Medical University

Weibing Miao

First Affiliated Hospital of Fujian Medical University

Shaobo Yao ( $\square$ yaoshaobo008@163.com)

The First Affiliated Hospital of Fujian Medical University https://orcid.org/0000-0003-2245-871X

\section{Research Article}

Keywords: Prostate cancer, 68Ga-DOTA-DiPSMA, 68Ga-PSMA-11, Dosimetry, PET/CT

Posted Date: August 24th, 2021

DOI: https://doi.org/10.21203/rs.3.rs-819948/v1

License: (1) (1) This work is licensed under a Creative Commons Attribution 4.0 International License. Read Full License 


\section{Abstract}

\section{Purpose}

This prospective trial aimed to evaluate the safety, dosimetry, biodistribution, and diagnostic efficacy of a novel theranostic probe ${ }^{68} \mathrm{Ga}$-DOTA-DiPSMA. Also, we have performed the first comparison with ${ }^{68} \mathrm{Ga}$ PSMA-11 in prostate cancer (PCa) patients.

\section{Methods}

Five healthy volunteers and ten PCa patients with a previous clinical ${ }^{68} \mathrm{Ga}-\mathrm{PSMA}-11 \mathrm{PET} / \mathrm{CT}$ were injected with an intravenous bolus of ${ }^{68} \mathrm{Ga}$-DOTA-DiPSMA with a dose of $1.85 \mathrm{MBq} / \mathrm{kg}$. Healthy volunteers received serial whole-body PET scans from the time of injection up to 60 min post-injection, with a second PET/CT scanning at 120 min post-injection. In PCa patients, low-dose CT scan, whole-body PET was performed with 2 min per bed position in 40 min post-injection. In addition, ${ }^{68} \mathrm{Ga}$-PSMA-11 scanning was performed on PCa patients within 10 days under the same acquisition procedure. Absorbed organ doses and effective doses were calculated using OLINDA/EXM. Normal organ uptake and tumor lesion uptake $\left(\mathrm{SUV}_{\max }\right)$ were measured. A lesion-by-lesion analysis was performed.

Results

${ }^{68} \mathrm{Ga}$-DOTA-DiPSMA administration was safe and well-tolerated. The kidneys received the highest absorbed dose (114.46 $\pm 29.28 \mathrm{uSv} / \mathrm{MBq})$, followed by the urinary bladder wall $(100.82 \pm 46.22$ $\mathrm{uSv} / \mathrm{MBq}$ ) in accordance with the expected PSMA renal excretion of the tracer. The mean effective dose was $19.46 \pm 1.73 \mu \mathrm{Sv} / \mathrm{MBq}$. The SUV $\mathrm{max}_{\text {max }}$ of ${ }^{68} \mathrm{Ga}$-PSMA-11 and ${ }^{68} \mathrm{Ga}$-DOTA-DiPSMA PET/CT for PCa lesions, bone metastases, and lymph node metastases were $11.2 \pm 10.76$ vs. $4.41 \pm 2.72,7.6 \pm 1.58$ vs. $2.95 \pm 1.11$ and $4.86 \pm 1.94$ vs. $3.26 \pm 1.2$, respectively.

\section{Conclusion}

Injection of ${ }^{68} \mathrm{Ga}$-DOTA-DiPSMA is safe and associated with low absorbed and effective doses. Compared to ${ }^{68} \mathrm{Ga}$-PSMA-11, ${ }^{68} \mathrm{Ga}$-DOTA-DiPSMA shows comparable pharmacokinetics and detection ability in PCa patients that warrant further head-to-head comparison. Low non-specific uptake in salivary glands and kidneys of ${ }^{68} \mathrm{Ga}$-DOTA-DiPSMA indicates potential radioligand therapy (RLT) application when labeled with ${ }^{177} \mathrm{Lu},{ }^{90} \mathrm{Y}$, or ${ }^{225} \mathrm{Ac}$.

\section{Introduction}

Prostate cancer $(\mathrm{PCa})$ is the most frequently diagnosed cancer in men and one of the lethal malignant diseases leading to male cancer-related death worldwide [1]. The accurate presence and location of primary or recurrent tumors are critical for planning effective patient management [2]. 
The diagnostic capability of conventional anatomic imaging such as MRI and CT to determine prostate cancer is limited in metastases and specificity [3]. Only prostate biopsy is the definitive way to confirm prostate cancer [1]. Multiple needle biopsy will increase the positive rate of lesions determination significantly. However, it is difficult to determine distant metastases and increase the risk of complications resulting from biopsy operation [1]. There has been an unmet need for more advanced imaging modalities to determine primary and metastatic lesions that can be helpful to prostate cancer patient management (observation, salvage local therapy, systemic therapy). PET with ${ }^{18} \mathrm{~F}$-FDG is effective for most malignant tumors, but it has insufficient specificity for prostate cancer and is prone to have false-positive results. Therefore, it is urgent to discover new nuclear medicine imaging agents with more specificity for prostate cancer.

Prostate-Specific Membrane Antigen (PSMA) is a transmembrane glycoprotein enzyme selectively overexpressed in prostate cancer cells, with its expression increasing in higher-grade malignancy [4]. PET imaging with PSMA probes targeting various prostate cancer-specific markers will provide additional molecular information to facilitate lesion detection and staging [5].

Recently, a relatively new nuclear imaging modality ${ }^{68} \mathrm{Ga}$-PSMA PET/CT imaging with good PCa diagnosis and staging performance, has become increasingly utilized to evaluate PCa aggressiveness, especially in patients with biochemical recurrence after surgery [6-8]. PSMA can be coupled with different chelators and labeled with corresponding radionuclides for different purposes. The most widely used PSMA ligands in clinical examination are PSMA-11 and PSMA-617 containing different linkers and chelators. According to the previously published papers, ${ }^{68} \mathrm{Ga}$-PSMA-11 and ${ }^{177}$ Lu-PSMA-617 are molecular-pair in metastatic castration-resistant PCa (mCRPC) diagnosis and radioligand therapy (RLT) $[9,10]$. However, considering the high nonspecific uptake in the salivary, kidney, and bone marrow of ${ }^{68} \mathrm{Ga}-$ PSMA-11 and ${ }^{177}$ Lu-PSMA-617, novel PSMA tracers with low accumulation in normal organs are urgently needed.

We have discovered a PSMA dimer (DOTA-DiPSMA, Fig. 1), and proved its imaging ability and low unspecific uptake (salivary, kidney) in preclinical experiments, which will be published later. In this study, we aimed to evaluate the safety, biodistribution and dosimetry of ${ }^{68} \mathrm{Ga}$-DOTA-DiPSMA in healthy volunteers. Furthermore, a head-to-head comparison of ${ }^{68} \mathrm{Ga}$-DOTA-DiPSMA vs. ${ }^{68} \mathrm{Ga}$-PSMA-11, including lesion detection rate, tumor uptake, and biodistribution in PCa patients were performed.

\section{Methods}

\section{Healthy Volunteers and Patients}

This study was approved by the Independent Ethics Committee of First Affiliated Hospital of Fujian Medical University (No. MRCTA, ECFAH of FMU[2019]293). All subjects gave written informed consent and were registered at ClinicalTrials.gov (NCT04525612). Five healthy volunteers and ten patients were enrolled (Table 1; Fig. 2). Five healthy volunteers (5 men, age range $42-76$ y mean age \pm SD, $59.83 \pm 11.65$ 
$y$ ]; weight range, $55.0-78.0 \mathrm{~kg}$ [mean weight $\pm S D, 70.27 \pm 13.05 \mathrm{~kg}$ ]) were enrolled to validate the safety, biodistribution and radiation dosimetry of ${ }^{68} \mathrm{Ga}$-DOTA-DiPSMA in this study. Exclusion criteria consisted of mental illness conditions, severe liver or kidney disease with serum creatinine greater than $3.0 \mathrm{mg} / \mathrm{dL}$ $(270 \mathrm{mM})$, or any hepatic enzyme level 5 times or more than the standard upper limit. Participants were also excluded if they were known to have severe allergy or hypersensitivity to intravenous radiographic contrast or claustrophobia during PET/CT scanning.

A total of 10 patients who were newly diagnosed as having prostate cancer by sextant core-needle biopsy and had not received any prior therapy were enrolled with written informed consent. The inclusion criteria were being between 40 and 80 years old, having a prostate neoplasm identified by ultrasound or MRI, being diagnosed by needle biopsy as having prostate cancer. The exclusion criteria included claustrophobia, kidney or liver failure, and inability to fulfill the study. The demographics of healthy volunteers and patients are listed in Table 1 . All the patients underwent ${ }^{68} \mathrm{Ga}-\mathrm{PSMA}-11 \mathrm{PET} / \mathrm{CT}$, and ${ }^{68} \mathrm{Ga}-$ DOTA-DiPSMA PET/CT for comparison within 1 week. MRI or enhanced CT and MDP (methylene diphosphonate) bone scintigraphy were performed within 2 weeks for comparison.

Subject age averaged $73.6 \pm 4.6$ years (median 74.5 years; range $67-80$ years), and body mass averaged $65.2 \pm 11 \mathrm{~kg}$ (median $65.4 \mathrm{~kg}$; range $51.8-92.4 \mathrm{~kg}$ ). Serum PSA values averaged $22.3 \pm 17.6 \mathrm{ng} / \mathrm{ml}$ (median $13.9 \mathrm{ng} / \mathrm{ml}$; range $6.27-60.1 \mathrm{ng} / \mathrm{ml}$ ). The reported serum PSA levels were the most recent clinical values at the time of ${ }^{68} \mathrm{Ga}$-PSMA imaging. The interval between ${ }^{68} \mathrm{Ga}$-PSMA-11 and ${ }^{68} \mathrm{Ga}$-DOTA-DiPSMA PET/CT imaging averaged $1.3 \pm 0.5$ days (median 1 day; range 1-2 days). Subjects are numbered chronologically in the order of imaging with ${ }^{68} \mathrm{Ga}-\mathrm{DOTA}$-DiPSMA.

\section{Safety Assessment}

Patient safety was assessed and graded according to Common Terminology Criteria for Adverse Events (version 5.0), electrocardiograms, physical examination, and vital signs (blood pressure, respiratory rate, heart rate, body temperature). Within the first $72 \mathrm{~h}$ after ${ }^{68} \mathrm{Ga}$-DOTA-DiPSMA injection, the research team kept phone contact with each subject monitoring their adverse event (AE) responses.

\section{Radiopharmaceutical Preparation}

Precursors were supplied by Prof. Cui from Key Laboratory of Radiopharmaceuticals, Ministry of Education, Beijing Normal University. ${ }^{68} \mathrm{Ga}^{3+}$ was eluted from a ${ }^{68} \mathrm{Ge} /{ }^{68} \mathrm{Ga}$ generator (JSC Isotope, Russian) using $0.1 \mathrm{M} \mathrm{HCl}$ and mixed with $1.25 \mathrm{M} \mathrm{NaOAc}$ buffer to adjust $\mathrm{pH}$ to around 4.0. Radiolabeling of DiPSMA-DOTA-COOH was performed in a sterile hot cell. The radiochemical purity of the product ${ }^{68} \mathrm{Ga}-$ DOTA-DiPSMA exceeded $99 \% .{ }^{68} \mathrm{Ga}-\mathrm{PSMA}-11$ was synthesized following a procedure reported previously [12].

\section{Examination Procedures}


For healthy volunteers, the blood pressure, pulse, respiratory frequency, and temperature were measured, and routine blood and urine tests, liver function, and renal function were examined immediately before and $24 \mathrm{~h}$ after the scan. In addition, any possible side effects during ${ }^{68} \mathrm{Ga}$-DOTA-DiPSMA PET/CT scanning and within 1 week after the examination were collected and analyzed. No specific subject preparation was requested on the day of ${ }^{68} \mathrm{Ga}$-DOTA-DiPSMA PET/CT. For the volunteers, after the wholebody low-dose CT scan, 111-222 MBq (3-6 mCi) of ${ }^{68} \mathrm{Ga}$-D OTA-DiPSMA were injected intravenously, followed by serial whole-body PET acquisitions. The whole body (from the top of the skull to the middle of the femur) of each volunteer was covered by 6 bed positions. The acquisition duration was $2 \mathrm{~min} / \mathrm{bed}$ position at $5,15,30,45,60$, and 120 min after injection.

For the patients, ${ }^{68} \mathrm{Ga}$-DOTA-DiPSMA PET/CT scanning was performed at 40 min after tracer administration. For each patient, $1.85 \mathrm{MBq}(0.05 \mathrm{mCi})$ of ${ }^{68} \mathrm{Ga}$-DOTA-DiPSMA per kilogram of body weight was injected intravenously. After a low-dose CT scan, whole-body PET was performed with 2 min per bed position (5- 6 bed positions depending on the patient's height). The emission data were corrected for randoms, dead time, scattering, and attenuation. The conventional reconstruction algorithm was used, and the images were zoomed with a factor of 1.2. The images were transferred to an MMWP workstation (Siemens) for analysis. All patients who were newly diagnosed with prostate cancer underwent ${ }^{68} \mathrm{Ga}$ PSMA-11 PET/CT for comparison within 1 week. For each patient, $1.85 \mathrm{MBq}(0.05 \mathrm{mCi})$ of ${ }^{68} \mathrm{Ga}-\mathrm{PSMA}-11$ per kilogram of body weight were injected intravenously. The imaging procedure and data analysis were the same as those for ${ }^{68} \mathrm{Ga}$-DOTA-DiPSMA PET/CT.

\section{Biodistribution Assessment and Dosimetry}

Image analysis was performed using MIM v6.9.4 (MIM Software Inc., Cleveland, Ohio, USA). VOIs were drawn over healthy organs on all ${ }^{68} \mathrm{Ga}$-DOTA-DiPSMA PET images, and SUVmean in these VOIs was determined to obtain the biodistribution of this tracer. Tumor lesions were evaluated in consensus by two nuclear medicine physicians.

All source organs with relevant detectable activity were delineated on the PET images with CT guidance for the healthy volunteers, using MIM software v6.9.4. Time-integrated activity coefficients (normalized cumulated activity; NCA) were calculated for each source organ by integrating their time-activity curves through curve fitting and normalizing the cumulated activity to the injected activity. Based on the timeintegrated activity coefficients, individual absorbed organ doses and the effective dose were determined using OLINDA/EXM v1.1 (Vanderbilt University, Nashville, Tennessee, USA). Calculations were performed with modeling of urinary bladder voiding. Parameters representing the fraction leaving the body via urine and biologic half-time were obtained from the fit and used to model urinary bladder voiding. Urinary bladder voiding models with voiding intervals of $1 \mathrm{~h}$ were applied. The 70-kg adult male models were used. Organ-absorbed doses, effective doses, and effective dose equivalents were calculated as mean \pm SD across subjects. 


\section{Results}

\section{Patient Safety}

${ }^{68} \mathrm{Ga}$-DOTA-DiPSMA was found to be safe and well-tolerated in all subjects. No adverse events or serious adverse events occurred after ${ }^{68} \mathrm{Ga}$-DOTA-DiPSMA injection for all the healthy volunteers and patients. No apparent changes in vital signs or clinical laboratory tests were found before and after the injection of ${ }^{68}$ Ga-DOTA-DiPSMA.

\section{Biodistribution}

Fig. 3, 4 illustrates the biodistribution of ${ }^{68} \mathrm{Ga}$-DOTA-DiPSMA as a function of time in healthy volunteers. The whole-body background of ${ }^{68} \mathrm{Ga}$-DOTA-DiPSMA was low. The highest uptake was observed in the kidney with a SUV mean $_{\text {of }} 43.4 \pm 26.8$ at 5 min p.i. and further decreased to $11.4 \pm 6.5$ at 120 min p.i. The spleen, liver, salivary gland, and small intestine showed moderate uptake, with SUV mean of $2.90 \pm 1.5,1.89 \pm 0.75,2.30 \pm 0.87$, and $2.42 \pm 0.64$ at 30 min after injection, respectively. Low background uptake was observed in the brain, lungs, muscle, red marrow, heart, thyroid, gall bladder, pancreas, stomach, bone, and large intestine. The rapid presence in the kidneys, followed by a passage toward the urinary bladder, illustrates the tracer's fast and mainly renal excretion.

\section{Dosimetry}

The average estimated absorbed organ in healthy volunteers was summarized in Table 2. The highest absorbed dose was received by the kidneys (114.46 $\pm 29.28 \mathrm{uSv} / \mathrm{MBq})$, followed by the urinary bladder wall $(100.82 \pm 46.22 \mathrm{uSv} / \mathrm{MBq})$. The mean effective dose was $19.46 \pm 1.73 \mu \mathrm{Sv} / \mathrm{MBq}$.

\section{Detection of Primary Prostate Cancer}

For the 10 patients with primary prostate cancer, ${ }^{68} \mathrm{Ga}$-DOTA-DiPSMA PET/CT showed 27 positive findings $囚$ including 16 prostate lesions, 4 bone metastases, 5 lymph node metastases and 2 seminal vesicle metastases. The primary lesions were confirmed by needle biopsy. SUV $\mathrm{max}_{\max }$ for prostate lesions, bone metastases, and lymph node metastases were $4.41 \pm 2.72,2.95 \pm 1.11$ and $3.26 \pm 1.2$, respectively. For ${ }^{68} \mathrm{Ga}$-PSMA-11 PET/CT, all lesions were positive, with the SUV $_{\max }$ of $11.2 \pm 10.76,7.6 \pm 1.58$, and 4.86 \pm 1.94 , respectively (Table 3, Fig. 5). Comparing normal tissues and organs background between ${ }^{68} \mathrm{Ga}$ DOTA-DiPSMA and ${ }^{68} \mathrm{Ga}$-PSMA-11 in Pca patients at 40 min p.i., significantly lower uptake was observed in the salivary glands (SUV $\max 4.88 \pm 2.04$ vs. $12.15 \pm 1.90, p=0.000)$, liver $(2.92 \pm 1.05$ vs.

$5.64 \pm 1.19, p=0.000)$, kidney $(23.40 \pm 11.27$ vs. $41.48 \pm 11.02, p=0.005)$ and spleen $(3.16 \pm 0.64$ vs. $7.78 \pm 1.99, p=0.000)$ in ${ }^{68}$ Ga-DOTA-DiPSMA, respectively (Table 4, Fig. 6).

\section{Discussion}


To our knowledge, this is the first human study to evaluate the novel tracer ${ }^{68} \mathrm{Ga}$-DOTA-DiPSMA in healthy volunteers and patients with PCas. This tracer is a new type of Ga-68-labeled dimer PSMA imaging agent with a simple structure, easy synthesis, and low synthesis cost. ${ }^{68} \mathrm{Ga}-\mathrm{DOTA}$-DiPSMA can be prepared by a one-step labeling reaction in high yield greater than $95 \%$ between ${ }^{68} \mathrm{Ga}^{3+}$ ions eluted from a germaniumgallium generator and the precursor DiPSMA-DOTA-COOH.

Here, we presented the results of an independently performed first clinical evaluation of ${ }^{68} \mathrm{Ga}$-DOTADiPSMA in five healthy volunteers, including biodistribution, dosimetry and safety. Also, we have performed the head-to-head lesion comparison of ${ }^{68} \mathrm{Ga}$-DOTA-DiPSMA to ${ }^{68} \mathrm{Ga}$-PSMA-11 in ten PCa patients.

The results showed that this tracer displayed favorable biodistribution, dosimetry features and welltolerated in all patients. Both ${ }^{68} \mathrm{Ga}$-DOTA-DiPSMA and ${ }^{68} \mathrm{Ga}$-PSMA-11 showed high PSMA affinity. The biodistribution of ${ }^{68} \mathrm{Ga}$-DOTA-DiPSMA was similar to that of ${ }^{68} \mathrm{Ga}$-PSMA-11. The highest uptake was observed in the kidney and rapidly cleared through urinary system in both tracers, consistent with the published ${ }^{68} \mathrm{Ga}$-PSMA-11 results [13-15]. ${ }^{68} \mathrm{Ga}$-DOTA-DiPSMA PET/CT images showed lower uptake in normal organs, including salivary glands, liver and spleen than ${ }^{68} \mathrm{Ga}-\mathrm{PSMA}-11$.

The dosimetry data of ${ }^{68} \mathrm{Ga}$-DOTA-DiPSMA showed a little lower yet comparable effective dose than ${ }^{68} \mathrm{Ga}-\mathrm{PSMA}-11$ (0.019 mSv/MBq vs. 0.022/0.023 mSv/MBq) [14,15], salivary glands $(0.024 \mathrm{mSv} / \mathrm{MBq}$ vs. $0.089 / \mathrm{mSv} / \mathrm{MBq})$, kidney (0.114 mSv/MBq vs. $0.240 \mathrm{mSv} / \mathrm{MBq})$, liver $(0.0240 \mathrm{mSv} / \mathrm{MBq}$ vs. $0.053 / \mathrm{MBq})$, spleen $(0.031 \mathrm{mSv} / \mathrm{MBq}$ vs. $0.046 \mathrm{mSv} / \mathrm{MBq})$, respectively [15]. We thought the lower liver and spleen dose of ${ }^{68} \mathrm{Ga}$-DOTA-DiPSMA might be attributed to the dosimetry methodology.

It is crucial to reduce the radiation dose of non-specific organs and tissues in the field of radionuclide therapy (RLT) [16]. ${ }^{177}$ Lu-PSMA-617 RLT is a promising option for patients with metastatic castrationresistant prostate cancer (mCRPC) [17-20]. Based on the lower non-specific uptake of ${ }^{68} \mathrm{Ga}$-DOTADiPSMA than ${ }^{68} \mathrm{Ga}$-PSMA-11. The radiation dosimetry in normal organs seemed to be reduced when DOTA-DiPSMA was labeled with ${ }^{177}$ Lu for RLT.

A critical finding of our study is the high tumor accumulation of ${ }^{68} \mathrm{Ga}-D O T A-D i P S M A$, which showed high tumor uptakes with the highest SUV $_{\max }$ up to 10.6 on ${ }^{68} \mathrm{Ga}$-DOTA-DiPSMA. The tumor lesion detection rate of ${ }^{68} \mathrm{Ga}$-DOTA-DiPSMA was the same as ${ }^{68} \mathrm{Ga}$-PSMA-11 in this pilot series of newly diagnosed prostate cancer patients. However, ${ }^{68} \mathrm{Ga}$-DOTA-DiPSMA showed lower uptake $\left(\mathrm{SUV}_{\text {max }}\right)$ in some lesions than ${ }^{68} \mathrm{Ga}$-PSMA-11, such as primary tumor ( $4.41 \pm 2.72$ vs. $\left.11.23 \pm 10.76\right)$, iliac lymph node $(3.26 \pm 1.20$ vs. $4.86 \pm 1.94)$, bone $(2.95 \pm 1.11$ vs. $7.62 \pm 1.58)$, and seminal vesicle $(3.95 \pm 2.61$ vs. $10.35 \pm 7.00)$, respectively. ${ }^{68} \mathrm{Ga}$-DOTA-DiPSMA seems to have a lower uptake in small lesions and distant metastasis [21-22]. However, the lower uptake of ${ }^{68} \mathrm{Ga}$-DOTA-DiPSMA in normal organs than ${ }^{68} \mathrm{Ga}-\mathrm{PSMA}-11$ maybe 
its advantage. The small lesion near the urinary bladder would be more apparent with this relatively low background.

The primary limitation of our study is the sample size which did not enable accurate multivariate regression analysis about comparing ${ }^{68} \mathrm{Ga}$-DOTA-DiPSMA to ${ }^{68} \mathrm{Ga}$-PSMA-11. Besides, neither blood nor urine samples were collected in our study, which will allow for the stability test in vivo. Further detailed and head-to-head comparison studies are required.

\section{Conclusion}

${ }^{68} \mathrm{Ga}$-DOTA-DiPSMA is safe and well-tolerated and shows favorable dosimetry and biodistribution in healthy volunteers and detection performances in PCa patients. Lesion detection rate and tumor-tobackground ratios were comparable to ${ }^{68} \mathrm{Ga}$-PSMA-11. The lower background of ${ }^{68} \mathrm{Ga}$-DOTA-DiPSMA showed its potential application for RLT when labeled with ${ }^{177} \mathrm{Lu}$. DOTA-DiPSMA is a promising novel theranostics tracer both for PCa patient diagnosis and RLT. Further validation by head-to-head comparisons in more extensive clinical trials is warranted.

\section{Declarations}

Funding information This study was funded in part by the National Natural Science Foundation of China (NSFC) 81971651, 82171982. Natural Science Foundation of Fujian 2019J01454, 2020J05249. Fujian Provincial Health Commission Science and Technology and Programme 2020GGA045.

Conflict of interest The authors declare that they have no conflict of interest.

Availability of data and materials Not applicable.

Code availability Not applicable.

Ethics approval All procedures involving human participants were carried out in accordance with the ethical standards of the institutional and/or national research committee and with the 1964 Helsinki Declaration and its later amendments or comparable ethical standards. This article does not contain any experiments with animals.

Consent to participate Informed consent was obtained from all individual participants included in the study.

ClinicalTrials.gov identifier: NCT04525612

\section{References}

1. Attard G, Parker C, Eeles RA, et al. Prostate cancer. Lancet. 2016 Jan 2;387(10013):70-82. doi: 10.1016/S0140-6736(14)61947-4. 
2. Mottet N, van den Bergh RCN, Briers E, et al. EAU-EANM-ESTRO-ESUR-SIOG Guidelines on Prostate Cancer-2020 Update. Part 1: Screening, Diagnosis, and Local Treatment with Curative Intent. Eur Urol. 2021 Feb;79(2):243-262. doi: 10.1016/j.eururo.2020.09.042.

3. Vos EK, Litjens GJ, Kobus T, et al. Assessment of prostate cancer aggressiveness using dynamic contrast-enhanced magnetic resonance imaging at 3 T. Eur Urol. 2013 Sep;64(3):448-55. doi: 10.1016/j.eururo.2013.05.045.

4. Bouchelouche K, Choyke PL, Capala J. Prostate specific membrane antigen- a target for imaging and therapy with radionuclides. Discov Med. 2010 Jan;9(44):55-61.

5. Perera M, Papa N, Roberts M, et al. Gallium-68 Prostate-specific Membrane Antigen Positron Emission Tomography in Advanced Prostate Cancer-Updated Diagnostic Utility, Sensitivity, Specificity, and Distribution of Prostate-specific Membrane Antigen-avid Lesions: A Systematic Review and Meta-analysis. Eur Urol. 2020 Apr;77(4):403-417. doi: 10.1016/j.eururo.2019.01.049.

6. Sachpekidis C, Kopka K, Eder M, et al. 68Ga-PSMA-11 Dynamic PET/CT Imaging in Primary Prostate Cancer. Clin Nucl Med. 2016 Nov;41(11):e473-e479. doi: 10.1097/RLU.0000000000001349.

7. Koerber SA, Utzinger MT, Kratochwil C, et al. ${ }^{68}$ Ga-PSMA-11 PET/CT in Newly Diagnosed Carcinoma of the Prostate: Correlation of Intraprostatic PSMA Uptake with Several Clinical Parameters. J Nucl Med. 2017 Dec;58(12):1943-1948. doi: 10.2967/jnumed.117.190314.

8. Wang B, Gao J, Zhang Q, et al. Diagnostic Value of ${ }^{68}$ Ga-PSMA PET/CT for Detection of Phosphatase and Tensin Homolog Expression in Prostate Cancer: A Pilot Study. J Nucl Med. 2020 Jun;61(6):873-880. doi: 10.2967/jnumed.119.236059.

9. Paganelli G, Sarnelli A, Severi S, et al. Dosimetry and safety of ${ }^{177}$ Lu PSMA-617 along with polyglutamate parotid gland protector: preliminary results in metastatic castration-resistant prostate cancer patients. Eur J Nucl Med Mol Imaging. 2020 Dec;47(13):3008-3017. doi: 10.1007/s00259020-04856-1.

10. Rahbar K, Ahmadzadehfar H, Kratochwil C, et al. German Multicenter Study Investigating 177LuPSMA-617 Radioligand Therapy in Advanced Prostate Cancer Patients. J Nucl Med. 2017 Jan;58(1):85-90. doi: 10.2967/jnumed.116.183194.

11. Sun M, Niaz MO, Nelson A, et al. Review of 177Lu-PSMA-617 in Patients With Metastatic CastrationResistant Prostate Cancer. Cureus. 2020 Jun 30;12(6):e8921. doi: 10.7759/cureus.8921.

12. Zamboglou C, Wieser G, Hennies S, et al. MRI versus $\triangle$ WGa-PSMA PET/CT for gross tumour volume delineation in radiation treatment planning of primary prostate cancer. Eur J Nucl Med Mol Imaging. 2016 May;43(5):889-897. doi: 10.1007/s00259-015-3257-5.

13. Chen $M$, Zhang Q, Zhang C, et al. Combination of ${ }^{68} \mathrm{Ga}-\mathrm{PSMA} P E T / C T$ and Multiparametric MRI Improves the Detection of Clinically Significant Prostate Cancer: A Lesion-by-Lesion Analysis. J Nucl Med. 2019 Jul;60(7):944-949. doi: 10.2967/jnumed.118.221010.

14. Afshar-Oromieh A, Hetzheim H, Kübler W, et al. Radiation dosimetry of (68)Ga-PSMA-11 (HBED-CC) and preliminary evaluation of optimal imaging timing. Eur J Nucl Med Mol Imaging. 2016 Aug;43(9):1611-20. doi: 10.1007/s00259-016-3419-0. 
15. Sandgren $K$, Johansson $L$, Axelsson J, et al. Radiation dosimetry of $\left[{ }^{68} \mathrm{Ga}\right] P S M A-11$ in low-risk prostate cancer patients. EJNMMI Phys. 2019 Jan 11;6(1):2. doi: 10.1186/s40658-018-0239-2.

16. Basic anatomical and physiological data for use in radiological protection: reference values. A report of age and gender-related differences in the anatomical and physiological characteristics of reference individuals. ICRP Publication 89. Ann ICRP. 2002;32(3-4):5-265.

17. Paganelli G, Sarnelli A, Severi S, et al. Dosimetry and safety of ${ }^{177}$ Lu PSMA-617 along with polyglutamate parotid gland protector: preliminary results in metastatic castration-resistant prostate cancer patients. Eur J Nucl Med Mol Imaging. 2020 Dec;47(13):3008-3017. doi: 10.1007/s00259020-04856-1.

18. Delker A, Fendler WP, Kratochwil C, et al. Dosimetry for (177)Lu-DKFZ-PSMA-617: a new radiopharmaceutical for the treatment of metastatic prostate cancer. Eur J Nucl Med Mol Imaging. 2016 Jan;43(1):42-51. doi: 10.1007/s00259-015-3174-7.

19. Kratochwil C, Giesel FL, Stefanova M, et al. PSMA-Targeted Radionuclide Therapy of Metastatic Castration-Resistant Prostate Cancer with 177Lu-Labeled PSMA-617. J Nucl Med. 2016 Aug;57(8):1170-6. doi: 10.2967/jnumed.115.171397.

20. Rasul S, Hacker M, Kretschmer-Chott E, et al. Clinical outcome of standardized ${ }^{177}$ Lu-PSMA-617 therapy in metastatic prostate cancer patients receiving $7400 \mathrm{MBq}$ every 4 weeks. Eur J Nucl Med Mol Imaging. 2020 Mar;47(3):713-720. doi: 10.1007/s00259-020-05082-5.

21. Afshar-Oromieh A, Avtzi E, Giesel FL, et al. The diagnostic value of PET/CT imaging with the (68)Galabelled PSMA ligand HBED-CC in the diagnosis of recurrent prostate cancer. Eur $\mathrm{J}$ Nucl Med Mol Imaging. 2015 Feb;42(2):197-209. doi: 10.1007/s00259-014-2949-6.

22. Fendler WP, Eiber M, Beheshti M, et al. ${ }^{68} \mathrm{Ga}$-PSMA PET/CT: Joint EANM and SNMMI procedure guideline for prostate cancer imaging: version 1.0. Eur J Nucl Med Mol Imaging. 2017 Jun;44(6):1014-1024. doi: 10.1007/s00259-017-3670-z.

\section{Tables}

Table 1. Summarizes the healthy volunteers and patient characteristics. 


\begin{tabular}{|lllll|}
\hline No & Age (year) & Sex & Weight (kg) & Inject dose \\
\hline HV1 & 55 & M & 73.8 & 3.3 \\
\hline HV2 & 42 & M & 92.3 & 5.5 \\
\hline HV3 & 67 & M & 60.2 & 3.8 \\
\hline HV4 & 56 & M & 74.3 & 5.44 \\
\hline HV5 & 50 & M & 65.9 & 5.5 \\
\hline 1 & 68 & $M$ & 51.8 & 3.6 \\
\hline 2 & 76 & $M$ & 67.2 & 3.0 \\
\hline 3 & 67 & $M$ & 61 & 4.1 \\
\hline 4 & 71 & $M$ & 92.4 & 3.05 \\
\hline 5 & 73 & $M$ & 67 & 3.06 \\
\hline 6 & 77 & $M$ & 65.1 & 3.85 \\
\hline 7 & 77 & $M$ & 57.9 & 2.70 \\
\hline 8 & 69 & $M$ & 65.6 & 2.98 \\
\hline 9 & 78 & $M$ & 56 & 2.8 \\
\hline 10 & 80 & $M$ & 68 & 2.8 \\
\hline
\end{tabular}

Table 2. Estimated absorbed organ doses and effective dose for ${ }^{68} \mathrm{Ga}$-DOTA-DiPSMA in healthy volunteers. 


\begin{tabular}{|c|c|c|}
\hline Absorbed dose & Mean (uSv/MBq) & SD (uSv/MBq) \\
\hline Adrenals & 14.44 & 0.34 \\
\hline Brain & 11.16 & 0.43 \\
\hline Breasts & 10.94 & 0.42 \\
\hline Gallbladder Wall & 13.12 & 3.33 \\
\hline LLI Wal & 12.47 & 3.71 \\
\hline Small Intestine & 12.36 & 3.61 \\
\hline Stomach Wall & 11.74 & 3.51 \\
\hline ULI Wall & 12.18 & 3.57 \\
\hline Heart Wall & 11.38 & 3.48 \\
\hline Kidneys & 114.46 & 29.28 \\
\hline Liver & 24.76 & 5.38 \\
\hline Lungs & 12.12 & 0.41 \\
\hline Muscle & 12.24 & 0.30 \\
\hline Ovaries & 14.26 & 0.13 \\
\hline Pancreas & 14.56 & 0.31 \\
\hline Red Marrow & 11.38 & 3.74 \\
\hline Osteogenic Cells & 14.53 & 5.46 \\
\hline Skin & 10.68 & 0.33 \\
\hline Spleen & 30.98 & 7.76 \\
\hline Testes & 12.38 & 0.13 \\
\hline Thymus & 12.14 & 0.48 \\
\hline Thyroid & 12.04 & 0.48 \\
\hline Urinary Bladder Wall & 100.82 & 46.22 \\
\hline Uterus & 15.54 & 0.61 \\
\hline Salivary glands & 24.32 & 7.11 \\
\hline Total Body & 9.71 & 4.93 \\
\hline Effective Dose Equivalent & 26.42 & 3.68 \\
\hline Effective Dose & 19.46 & 1.73 \\
\hline
\end{tabular}


Table 3. Comparison of PET findings with ${ }^{68} \mathrm{Ga}$-DOTA-DiPSMA and ${ }^{68} \mathrm{Ga}-\mathrm{PSMA}-11$ in the initial ten subject study of ${ }^{68} \mathrm{Ga}$-DOTA-DiPSMA in prostate cancer patients with newly diagnosed. 


\begin{tabular}{|c|c|c|c|c|c|c|c|}
\hline \multirow[t]{2}{*}{ Subject } & \multirow[t]{2}{*}{ Age } & \multirow[t]{2}{*}{$\begin{array}{l}\text { Serum PSA } \\
\text { (ng/ml) }\end{array}$} & \multicolumn{2}{|c|}{$\begin{array}{l}\text { Site(s) of recurrence } \\
\text { detected by }\end{array}$} & \multicolumn{2}{|c|}{$\begin{array}{l}\text { SUV }_{\text {max }} \text { values for } \\
\text { detected lesions }\end{array}$} & \multirow[t]{2}{*}{$\begin{array}{l}\text { Lesion } \\
\text { location }\end{array}$} \\
\hline & & & PSMA-11 & DiPSMA & PSMA-11 & DiPSMA & \\
\hline \multirow[t]{5}{*}{1} & 68 & 26.95 & yes & yes & 7.4 & 3.3 & prostate \\
\hline & & & yes & yes & 6.0 & 1.9 & $\begin{array}{l}\text { T8 } \\
\text { vertebrae }\end{array}$ \\
\hline & & & yes & yes & 9.6 & 4.1 & Sacrum \\
\hline & & & yes & yes & 6.8 & 3.7 & Iliac \\
\hline & & & yes & yes & 8.1 & 2.1 & Iliac \\
\hline \multirow[t]{2}{*}{2} & 76 & 10.5 & yes & yes & 5.6 & 2.9 & prostate \\
\hline & & & yes & yes & 4.1 & 2.6 & prostate \\
\hline \multirow[t]{3}{*}{3} & 67 & 10.3 & yes & yes & 5.9 & 1.9 & prostate \\
\hline & & & yes & yes & 2.9 & 1.7 & prostate \\
\hline & & & yes & yes & 4.0 & 3.7 & prostate \\
\hline \multirow[t]{2}{*}{4} & 71 & 9.56 & yes & yes & 18.9 & 6.4 & prostate \\
\hline & & & yes & yes & 4.5 & 3.8 & prostate \\
\hline 5 & 73 & 8.62 & yes & yes & 20.4 & 4.0 & prostate \\
\hline \multirow[t]{2}{*}{6} & 77 & 6.27 & yes & yes & 3.1 & 2.1 & prostate \\
\hline & & & yes & yes & 2.6 & 2.3 & prostate \\
\hline 7 & 77 & 17.22 & yes & yes & 14.5 & 9.76 & prostate \\
\hline \multirow[t]{4}{*}{8} & 69 & 37.9 & yes & yes & 44.9 & 10.6 & prostate \\
\hline & & & yes & yes & 13.3 & 3.0 & prostate \\
\hline & & & yes & yes & 15.3 & 5.8 & $\begin{array}{l}\text { Seminal } \\
\text { vesicle }\end{array}$ \\
\hline & & & yes & yes & 5.4 & 2.1 & $\begin{array}{l}\text { Seminal } \\
\text { vesicle }\end{array}$ \\
\hline 9 & 78 & 35.72 & yes & yes & 14 & 6.6 & prostate \\
\hline \multirow[t]{3}{*}{10} & 80 & 60.1 & yes & yes & 13.5 & 5.9 & prostate \\
\hline & & & yes & yes & 7.3 & 2.6 & $\begin{array}{l}\text { lliac lymph } \\
\text { node }\end{array}$ \\
\hline & & & yes & yes & 4.4 & 3.1 & $\begin{array}{l}\text { lliac lymph } \\
\text { node }\end{array}$ \\
\hline
\end{tabular}




\begin{tabular}{|cccccc|}
\hline yes & yes & 4.1 & 4.6 & $\begin{array}{l}\text { Nliac lymph } \\
\text { node }\end{array}$ \\
\hline yes & yes & 6.2 & 4.3 & $\begin{array}{l}\text { Iliac lymph } \\
\text { node }\end{array}$ \\
\hline yes & yes & 2.3 & 1.7 & $\begin{array}{l}\text { \|liac lymph } \\
\text { node }\end{array}$ \\
\hline
\end{tabular}

Table 4. Comparison of normal organs SUV max $_{\text {with }}{ }^{68}$ Ga-DOTA-DiPSMA and ${ }^{68}$ Ga-PSMA-11

\begin{tabular}{|c|c|c|c|}
\hline & \multicolumn{2}{|l|}{$\begin{array}{l}\mathrm{SUV}_{\text {max }} \\
(\text { Mean } \pm \mathrm{SD})\end{array}$} & \multirow[t]{2}{*}{$P$-value } \\
\hline & PSMA-11 & DiPSMA & \\
\hline Thyriod & $1.62 \pm 0.50$ & $1.12 \pm 0.21$ & $0.013^{*}$ \\
\hline Brain & $0.08 \pm 0.05$ & $0.07 \pm 0.04$ & 0.364 \\
\hline Lung & $0.48 \pm 0.12$ & $0.40 \pm 0.09$ & 0.151 \\
\hline Thymus & $0.66 \pm 0.16$ & $0.53 \pm 0.19$ & 0.257 \\
\hline Heart bladder & $1.13 \pm 0.35$ & $1.18 \pm 0.21$ & 0.762 \\
\hline Heart content & $1.53 \pm 0.35$ & $1.94 \pm 0.50$ & $0.041^{*}$ \\
\hline Liver & $5.64 \pm 1.19$ & $2.92 \pm 1.05$ & $0.000^{*}$ \\
\hline Kidney & $41.48 \pm 11.02$ & $23.40 \pm 11.27$ & $0.005^{*}$ \\
\hline Stomach & $2.36 \pm 2.60$ & $0.99 \pm 0.57$ & $0.049^{*}$ \\
\hline Adrenal gland & $3.34 \pm 2.32$ & $1.76 \pm 0.64$ & $0.016^{*}$ \\
\hline Pancreas & $2.80 \pm 2.29$ & $1.48 \pm 0.43$ & $0.004^{*}$ \\
\hline Spleen & $7.78 \pm 1.99$ & $3.16 \pm 0.64$ & $0.000^{*}$ \\
\hline Gallbladder & $2.19 \pm 1.11$ & $0.97 \pm 0.41$ & $0.002^{*}$ \\
\hline Large intestine & $1.29 \pm 0.23$ & $0.92 \pm 0.33$ & $0.028^{*}$ \\
\hline Small intestine & $7.18 \pm 5.38$ & $2.26 \pm 1.70$ & $0.028^{*}$ \\
\hline Red marrow & $0.59 \pm 0.24$ & $0.44 \pm 0.20$ & 0.186 \\
\hline Muscle & $0.51 \pm 0.16$ & $0.65 \pm 0.24$ & 0.406 \\
\hline Salivary gland & $12.15 \pm 1.90$ & $4.88 \pm 2.04$ & $0.000^{*}$ \\
\hline
\end{tabular}

${ }^{*} P \otimes 0.05$. 
Figures

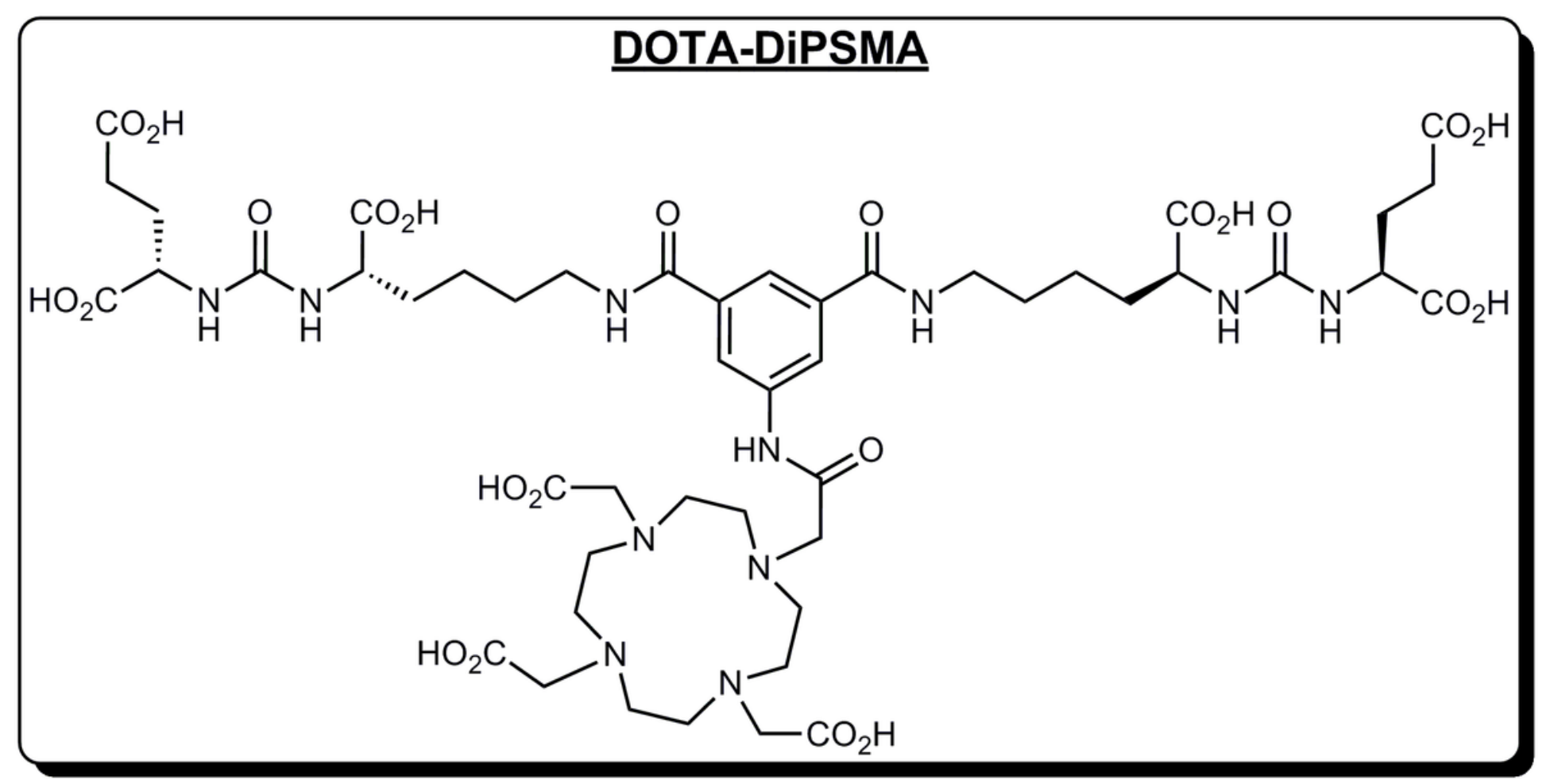

Figure 1

Structure of DOTA-DiPSMA. 


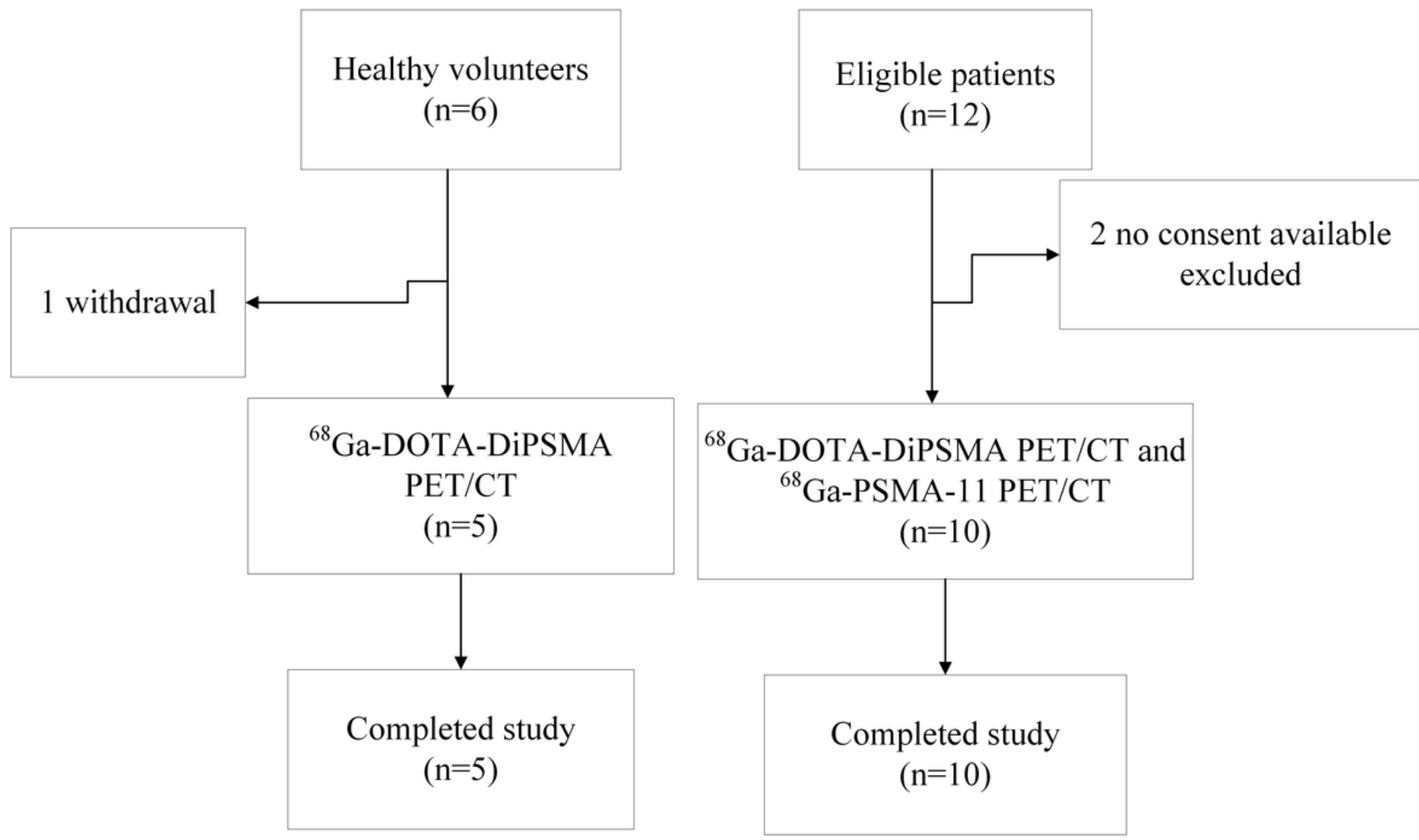

Figure 2

Study flowchart with excluded healthy volunteers and patients.

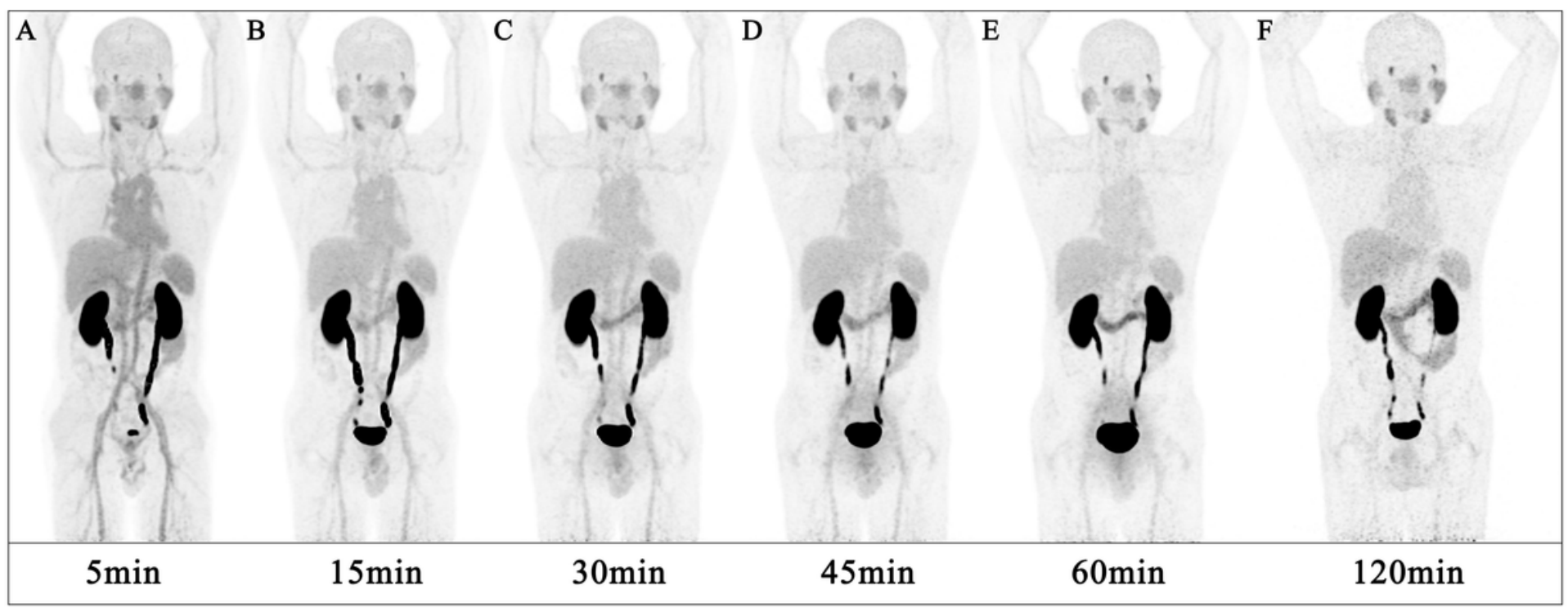

Figure 3 
Biodistribution of $68 \mathrm{Ga}$-DOTA-DiPSMA in healthy volunteers. A maximum intensity projection PET images at several time points post-injection of a 56-year-old male healthy volunteer.

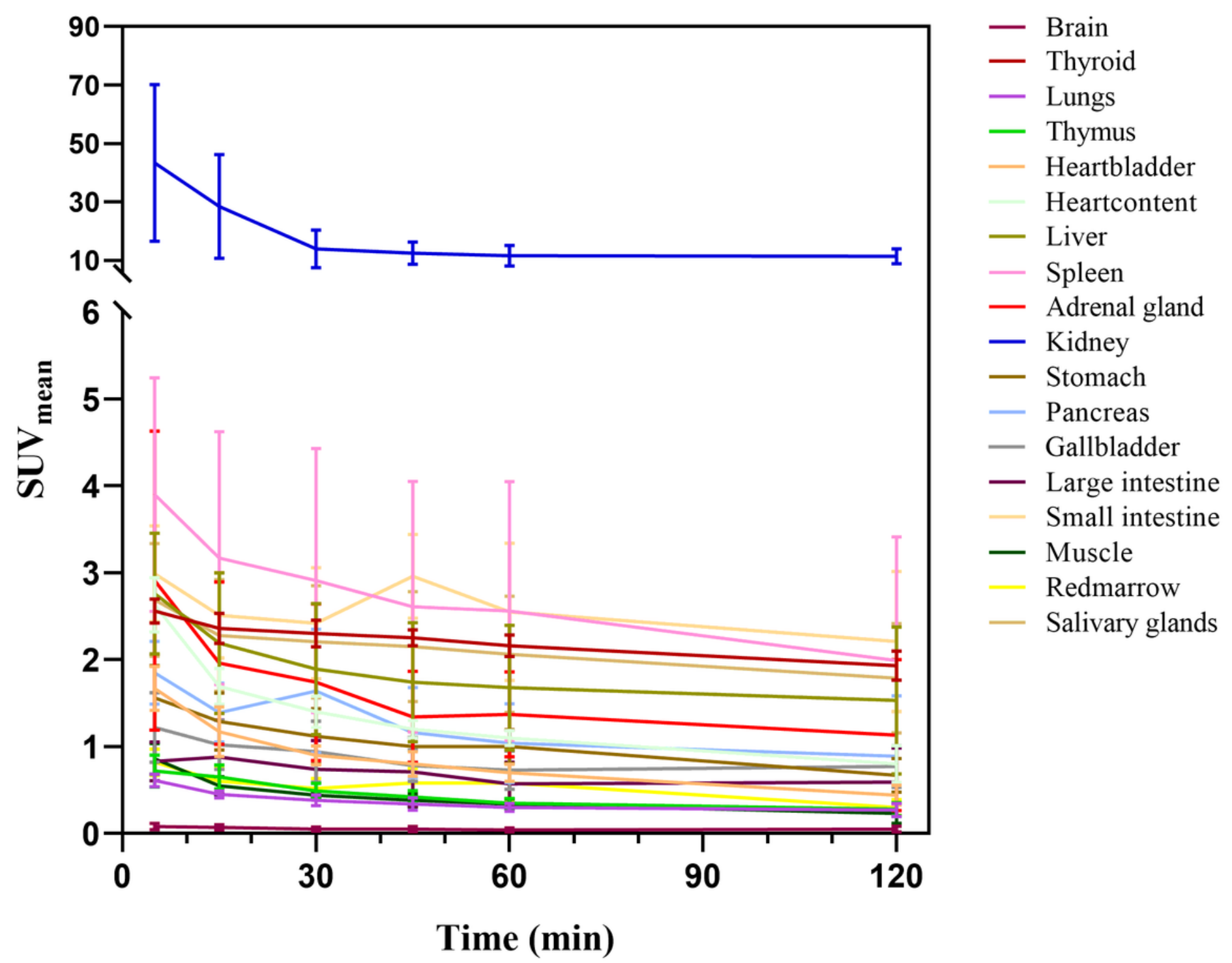

Figure 4

Mean SUVmean as a function of time for all healthy volunteers. 


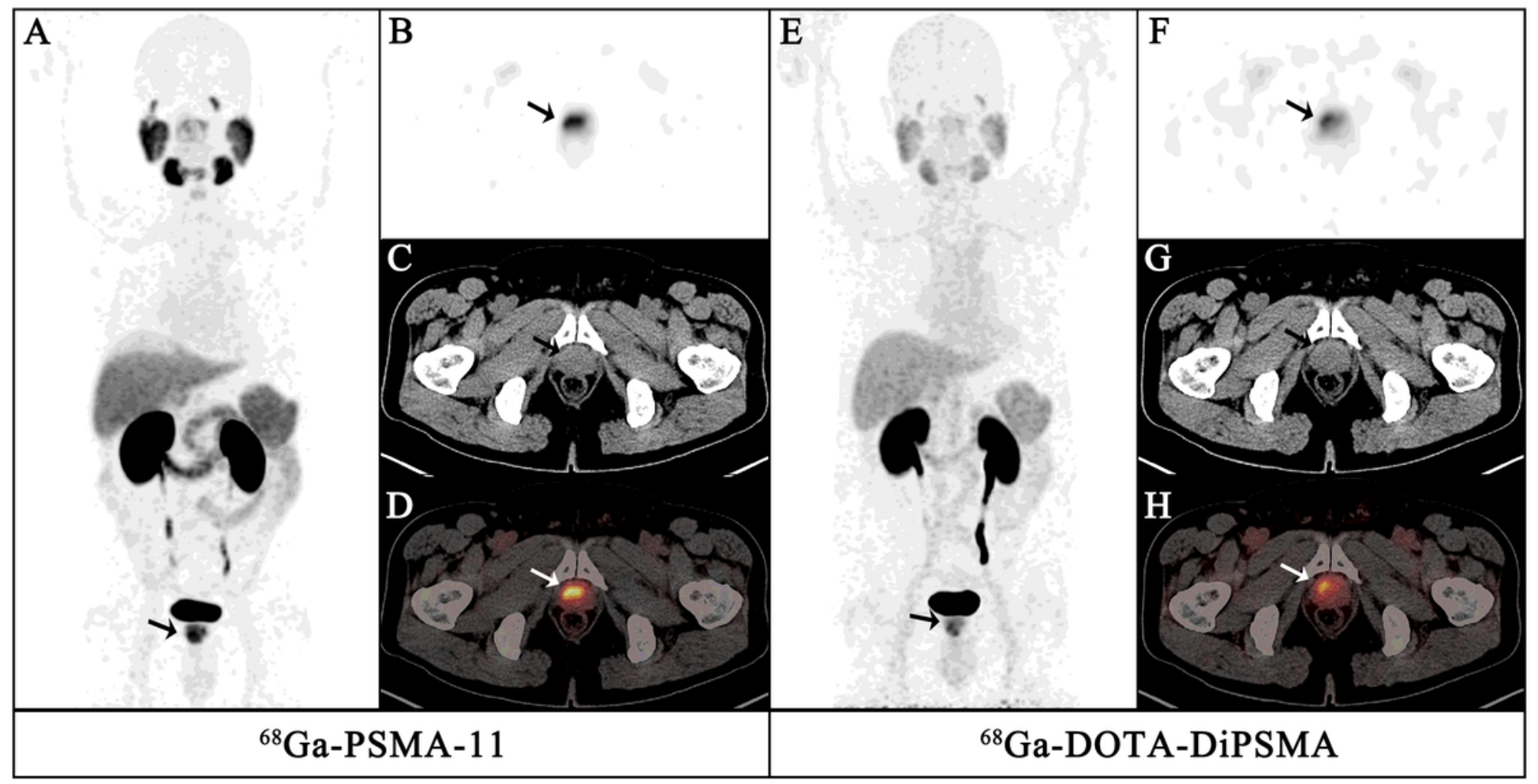

Figure 5

A 78-year-old-man confirmed prostate cancer for 1 week. The MIP of 68Ga-PSMA-11 PET/CT (A, arrows) showed significantly intense uptake in the prostate. Axial views of the prostate (B, PET; C, CT; D, fusion image) showed intense uptake (SUVmax 14) in the isodense nodule of the prostate (arrows). In 68GaDOTA-DiPSMA PET/CT (E, MIP; F, PET; G, CT; H, fusion image), the lesion showed moderate increased uptake of the tracer (SUVmax 6.6). 


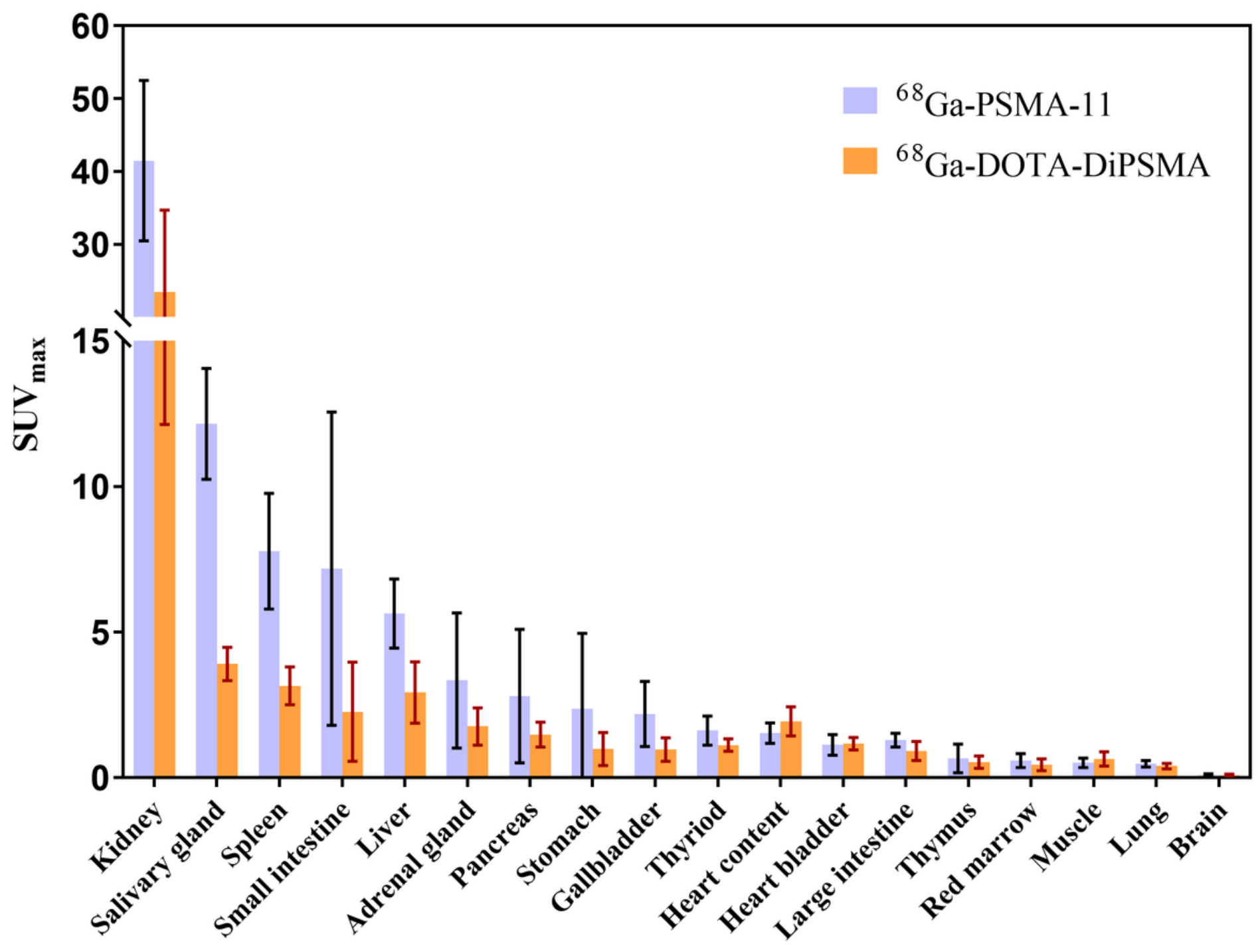

Figure 6

Normal organs SUVmax (Average in 10 patients) of 68Ga-DOTA-DiPSMA as compared with 68Ga-PSMA11 